\title{
Orbital Lymphangioma-Case Report and Review of the literature
}

\author{
Medina Andrade Abraham Alejandro MD¹, Estephania Feria Anzaldo MD¹, Ortiz Ramirez \\ Grecia Yael MD ${ }^{1}$, Clarisa Esther del Hierro Gutierrez MD ${ }^{1}$, Davila Avila Ned Merari MD ${ }^{1}$, \\ Vianhi G. López Rioja MD ${ }^{1}$, Luis Angel Medina Andrade $\mathbf{M D}^{2}$ \\ ${ }^{1}$ Oculoplastic Department, Association to Prevent Blindness in Mexico, Dr. Luis Sánchez Bulnes Hospital, \\ Vicente García Torres \# 46, San Lucas Coyoacán, 04030. Mexico City \\ ${ }^{2}$ General Surgery Department, General Zone Hospital 1A, Mexican Institute of Social Security, México City
}

*Corresponding Author: Luis Angel Medina Andrade, General Surgery Department, General Zone Hospital 1A, Mexican Institute of Social Security, Municipio Libre 270, Portales Norte, P.C. 03300, México City. Email: buismedina_5@hotmail.com

\section{INTRODUCTION}

Lymphangioma is a benign tumor of the lymphatic system, primarily seen within the first decade of life. It is classified by the International Society for the Study of Vascular Anomalies as a low-flow vascular malformation ${ }^{1}$. It occurs in $4 \%$ of all orbital lesions and $25 \%$ of the vascular lesions ${ }^{2,3}$.

Clinically they can be asymptomatic or manifests with unilateral proptosis, periocular edema, spontaneous or traumatic hemorrhage, ocular motility limitations and visual acuity changes. In childhood, it can typically present as a sudden proptosis after an upper respiratory infection or minor trauma. Visual loss can be due to compressive optic neuropathy when the hemorrhage is massive or the tumor itself compresses the optic nerve so we must stay aware about this pathology in the first stages of develope ${ }^{4}$.
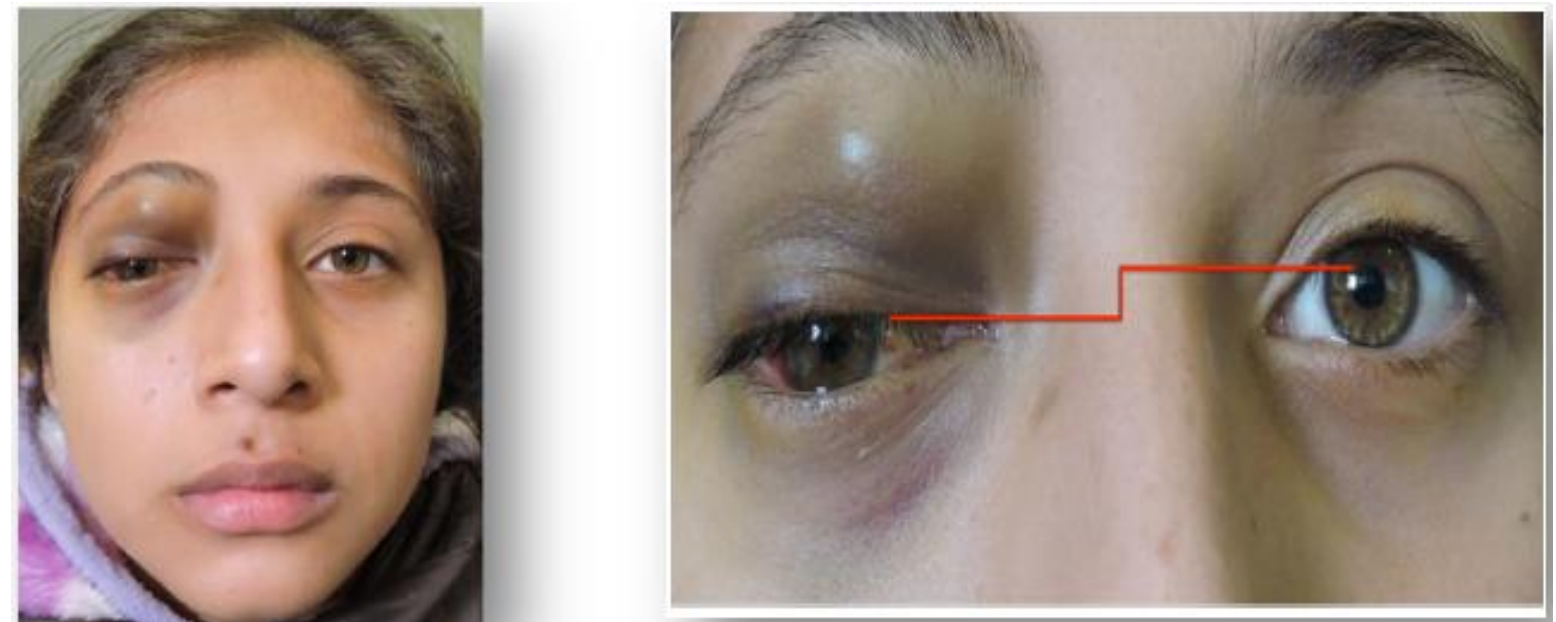

Fig1. Initial exploration. Right upper eyelid with ecchymosis and inferior displacement of eyeball

\section{CASE REPORT}

A 12-year-old female patient was referred to our center due to upper eyelid ecchymosis and midupper right eyelid lesion which displaces the eyeball towards inferior (Fig. 1), with a 20/50 visual acuity in the right eye, the lesion was not pulsatile, did not increase its size with Valsalva Maneuver, was not adhered to deep tissues, do not increase local temperature; it was producing a $3 \mathrm{~mm}$ hypoglobus and an exotropy of approximately 15 degrees on the right side. Ocular motility testing showed limitation of supraduction (-3) as well as adduction (-2), presenting vertical diplopia in supraduction and horizontal diplopia in adduction (Fig 2). The patient had a $3 \mathrm{~mm}$ protrusion of the right eye (Fig.3). She also presented conjunctival hemorrhage on the temporal side of the right eye, as well as ingurgitation of the episcleral vessels. The rest of ophthalmic examination was normal. 

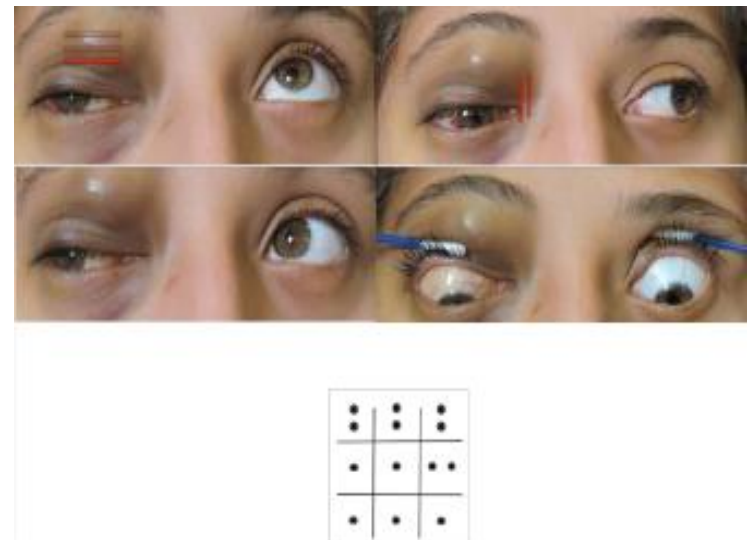

Fig2. Ocular motility testing with limitation of supraduction (-3) and adduction (-2), presenting vertical diplopia in supraduction and horizontal diplopia in adduction

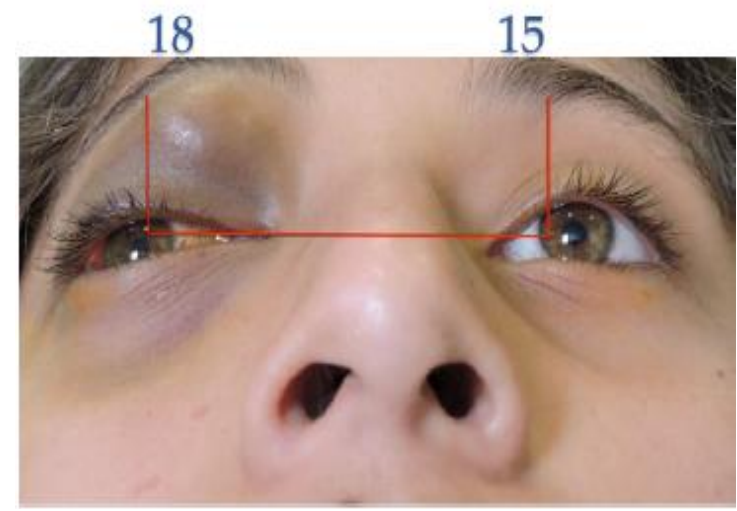

Fig3.Exophthalmometry with evidence of $18 \mathrm{~mm}$ in right eye and 15 in left eye, showing a non-axial proptosis

Computerized tomography (CT) of the orbits shows a multilobulated heterogeneous mass, with well-defined borders, without indentation of the eye globe, which extends from the equator to the ceiling of the orbit, without bone erosion, localized intra and extra-conal. In CT coronal sections, an occupational mass can be seen with an apparent capsule (Fig 4). The mass shows no contrast enhancement.

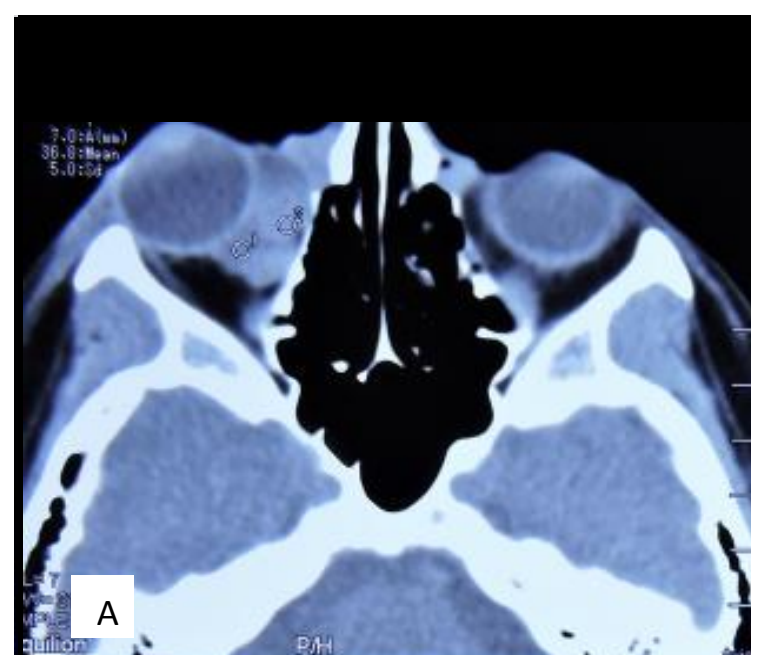

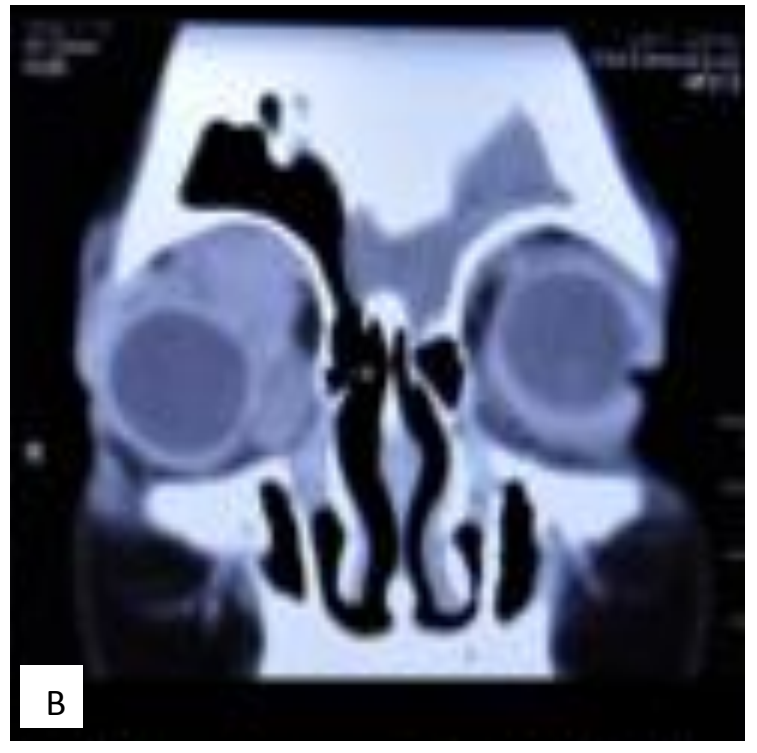

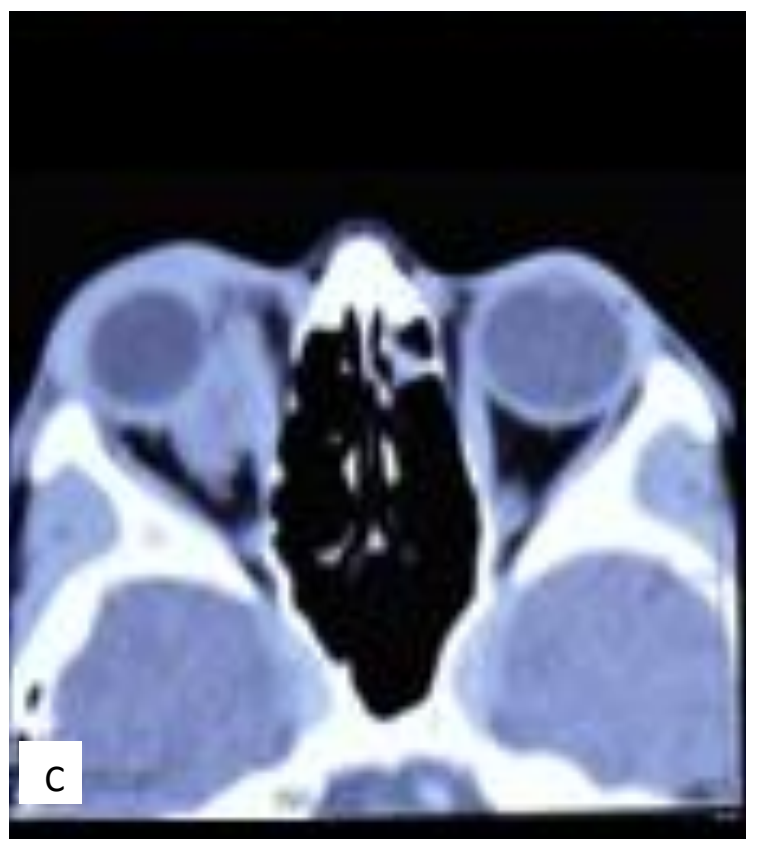

Fig4. CT scan showing well-defined, heterogeneous, multilobulated, encapsulated and no enhancing tumor.

Laboratory test (blood chemistry, blood count, coagulation and urinalysis) were normal. An excisional biopsy was performed, a deep violetcolored lesion was found, which, after aspiration gave a $8 \mathrm{ml}$ chocolate like liquid. Intralesional infiltrate of Bleomycin $(4 \mathrm{ml})$ was performed in the lesion. The superficial bag was removed and sent for histopathological examination. Pathologic findings revealed lymphatic channels, inflammatory infiltrate, hemosiderin deposit caused by excessive red blood cell destruction and a monolayer of flattened endothelial cells, making the diagnosis of Lymphangioma (Fig. 5). 


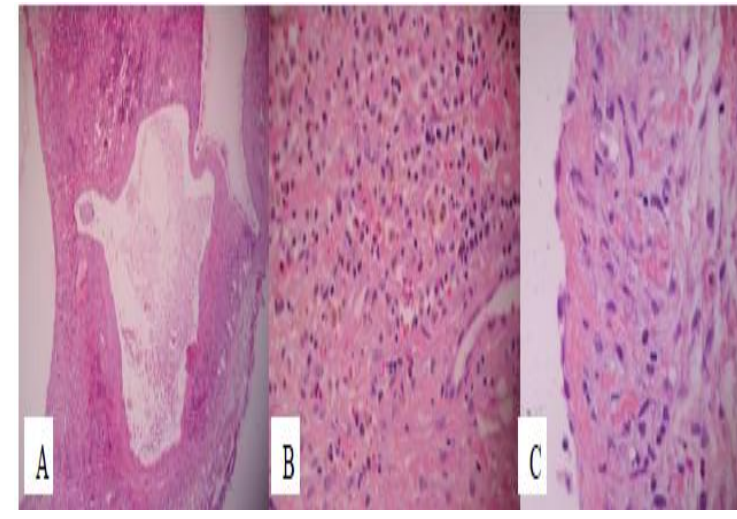

Fig.5. (A) Photomicrography 40x of HE tinction showing dilated lymphatic channels with peripheral connective tissue. (B) 100x photomicrography with clusters of lymphocytes and hemosiderophages for old hemorrhage $(C)$ 100x photomicrography showing thethick walls of a vessel of neoformation

At the 6-month follow-up there were no signs of relapse, with visual acuity recovered (Fig 6).

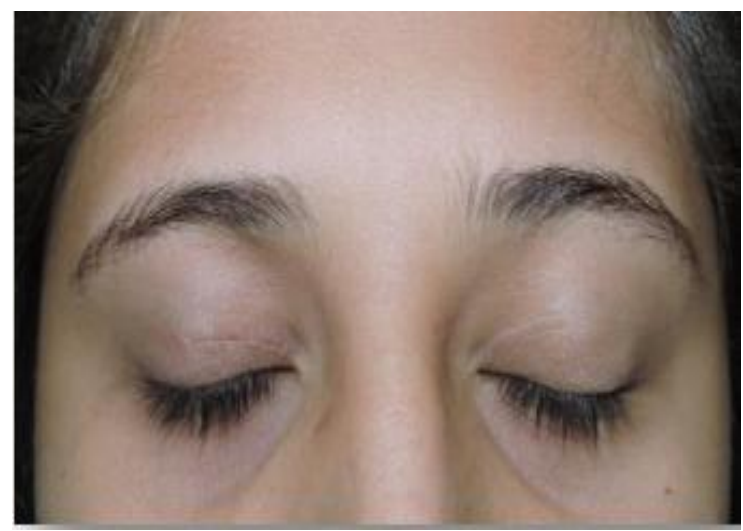

Fig6. Patient at 6-months follow-up

\section{DISCUSSION}

Some tracing experiments in endothelial lineage have confirmed the embryonic vein origin of lymphatic endothelium and that the gen Prox1 may function as the master regulator for lymphatic system, by reprogramming endothelial cells from blood vascular endothelial to lymphatic endothelial, allowing primary lymphatic plexus formation mediated by endothelial cell specific growth factor (VEGFC). As the orbit does not normally have lymphatics, therefore is theorized that orbital lymphatico-venous malformations are originated fromprimary orbital varices or from lymphatic or venous systems of the periorbital structures ${ }^{5}$.

Diagnosis is established with clinical features and imaging studies, including orbital ultrasonography, CT scan and MRI. In the orbital ultrasonography we can find an irregular shape lesion, poorly outlined, without increasing in size during Valsalva maneuver and typically appearing to infiltrate adjacent fat and muscle tissue. In A mode it shows spaces with low reflectivity and septae with highreflectivity ${ }^{6}$. In an orbital CT scan we can observe irregular heterogeneous masses that are not confined by fascial planes, they can show contrast enhancement and phlebeloids (calcified thrombi) due to intralesional hemorrhage ${ }^{7}$. In the orbital MRI the lesion presents increased signal intensity on $\mathrm{T} 2$ weighted images and cystic spaces with rim enhancement and bony over growth ${ }^{7}$. MRI provide superiority over CT in providing soft tissue detail without using ionizing radiation, and in areas surrounded by bone. Magnetic resonance imaging also can produce images in multiple planar orientations without the reformatting required by CT. Magnetic resonance imaging has superior capabilities to CT in these respects because its image production is unrelated to conventional $\mathrm{x}$ ray attenuation, but is dependent on electromagnetic characteristics of hydrogen nuclei $^{7,8}$. The details of magnetic resonance are complex and have been extensively reviewed in the radiology literature. The development of the MRI contrast agent gadolinium-DTPA has heightened the value of MRI in fully evaluating several ophthalmic entities. The effect of the paramagnetic contrast agent is to decrease the $\mathrm{T}$ 1 relaxation time, thus increasing the signal intensity of T1-weighted images. For all the previous mentioned facts, in the study of orbital lymphangioma the MRI is the study of choice by their unequalled differentiation of hemorrhagic cysts, and its ability to detect tumor feeding vessels ${ }^{8}$.

Current imaging methods allow non invasive diagnosis in almost all cases, however we can confirm the diagnosis by histopathology study, where we can find an unencapsulated lesion, irregular dilated lymphatic vessels that are coated in a monolayer of endothelial cells, separated by fibrous septae and dilated vascular vessels $^{9}$. Most of them presents evidence of old hemorrhageby the presence of cholesterol clefts, hemosiderin, lysed blood cells, lipid laden macrophages and foreign body reaction. Stromal components like smooth muscle fibers and loose connective tissue septa are present in varying degrees $^{10}$.

Despite being benign tumors, these lesions intrinsically invade surrounding tissue, complicating their treatment. The therapeutic options available are observation, decompression, $\mathrm{CO} 2$ laser therapy, systemic steroids and surgical excision ${ }^{11}$. Surgical 
resolution is difficult and usually there is residual tumor and recurrences, furthermore normal tissue can be damaged, thus surgical excision is not recommended as the first line treatment, it can be reserved to acute hemorrhagic episodes with optic nerve compression". There is no definitive treatment, although there are several sclerotherapy options as a new line of treatment to reduce recurrence rates or in unresectable cases. Some of these agents include OK 432, sodium tetradecylsulfate, sodium morrhuate and bleomycin, the latter being the preferred agent in the last couple of years ${ }^{11-13}$. Bleomycin, a glucopeptide produced by Streptomyces Verticilus, which acts by breaking DNA chains with free radicals, affects tumor cells. Its side effects are erythema, edema, pain, local necrosis and flagellate hyperpigmentation (resolves with drug interruption) ${ }^{14}$.

In general, traditional surgical treatment of these lesions is challenging, and patients may experience a prolonged and recurrent course. Because of potential damage to adjunct structures, surgical options can lead to morbidity. As noted, there are short comings in the published studies describing different treatments for orbital lymphaticovenous malformations. Inconsistent data, incomplete hemodynamic classification or description, small sample sizes, and lack of within-study comparisons with a control group and other treatments limit our ability to develop evidencebased treatment guidelines for these lesions ${ }^{5}$.

Orbital lymphaticovenous malformations tend to regrow after treatment. In fact, as described, it is not possible in many lesions to destroy or remove every endothelial cell, and the remaining mass of vessels and stroma can regrow over varying time periods because of residual hemodynamic forces. It is therefore more appropriate to use the term recrudescence instead of recurrence. The recrudescence rate of these lesions in other parts of the body can be as high as $40 \%$ after incomplete excision and $17 \%$ after macroscopically complete excision; however, the size of lymphaticovenous malformations after recrudescence is usually smaller than the initial lesion ${ }^{5}$.

The prognosis in patients is good overall, given the fact that it is a benign tumor with high risk of recurrence we have to inform the patients on clinical signs such as proptosis, strabismus, low visual acuity and pain for opportune evaluation.

\section{Conclusions}

Lymphangioma is a benign orbital tumor primarily seen within the first decade of life. MRI is the imaging study of choice for orbital lymphangioma. Due to its infiltrative nature, complete surgical resection is almost impossible in many cases, and postoperative recurrence is common. Intralesional injection of sclerosing agents is considered the first line of treatment.

\section{REFERENCES}

[1] ISSVA Classification of Vascular Anomalies (C2014 International Society for the Study of Vascular Anomalies Available at "issva.org/classification" Accessed July, 2017.

[2] Shields JA, Shields CL, Scartozzi R. Survey of 1264 patients with orbital tumors and simulating lesions: the 2002 Montgomery Lecture, Part 1. Ophthalmology 2004; 111:9971008.

[3] Bonavolontà G, Strianese D, Grassi P, et al. An analysis of 2,480 space-occupying lesions of the orbit from 1976 to 2011. OphthalPlastReconstr Surg. $2013 \quad$ MarApr;29(2):79-86.

[4] Young J, Chang Y, Bradford S, Jin S. Orbital Lymphangioma: Characteristics and Treatment Outcomes of 12 Cases. Korean J Ophthalmol. 2017; 31(3): 194-201.

[5] Nassiri N, Rootman J, Rootman DB, et al. Orbital lymphaticovenous malformations: current and future treatments. SurvOphthalmol 2015; 60:383.

[6] Ko F, DiBernardo CW, Oak J, et al. Confirmation of and differentiation among primary vascular lesions using ultrasonography. OphthalPlastReconstr Surg. 2011;27:431

[7] Bisdorff A, Mulliken J, Carrico J, et al. Intracranial vascular anomalies in patients with periorbital lymphatic and lymphaticovenous malformations. AJNR Am J Neuroradiol. 2007; 28(2): 335-41

[8] Bond J, Haik B, Taveras J, et al. Magnetic resonance imaging of orbital lymphangioma with and without gadolinium contrast enhancement. Ophthalmology. 1992; 99 (8):1318-24

[9] Harris G, Sakol P, Bonavolontà G, De Conciliis C. An analysis of thirty cases of orbital lymphangioma. Pathophysiologic considerations and management recommendations. Ophthalmology. 1990; 97(12):1583-92.

[10] Graeb D, Rootman J, Robertson W, et al. Orbital lymphangiomas: clinical, radiologic, and pathologic characteristics. Radiology. 1990; 175 (2): 417-21. 
[11] Schwarcz R, Ben Simon G, Cook T, Goldberg R. Sclerosing therapy as first line treatment for low vascular lesions of the orbit. Am J Ophthalmol. 2006; 141(2):333-9.

[12] Patel K, Kalantzis G, El-Hindy N, Chnag B. Sclerotherapy for Orbital Lymphangioma Case Series and Literature Review. In Vivo. 2017 ; 31(2): 263-266.

[13] Barnacle A, Theodorou M, Maling S, AbouRayyah Y. Sclerotherapy treatment of orbital lymphatic malformations: a large single-center experience. $\mathrm{Br} \mathrm{J}$ Ophthalmol. 2016; 100(2):204-8.

[14] Chen W, Huang Z, Chai Q, et al. Percutaneous sclerotherapy of massive macrocystic lymphatic malformations of the face and neck using fibrin glue with OK-432 and bleomycin. Int J Oral Maxilofac Surg. 2001; 40(6): 572-6.

Citation: Medina Andrade Abraham Alejandro et al. Orbital Lymphangioma-Case Report and Review of the literature. ARC Journal of Surgery.2017; 3(2):8-12.doi:dx.doi.org/10.20431/2455-572X.0302003.

Copyright: (C) 2017 Authors. This is an open-access article distributed under the terms of the Creative Commons Attribution License, which permits unrestricted use, distribution, and reproduction in any medium, provided the original author and source are credited. 